\title{
LÍNEA DE COSTA Y SISTEMAS DE INFORMACIÓN GEOGRÁFICA: MODELO DE DATOS PARA LA CARACTERIZACIÓN Y CÁLCULO DE INDICADORES EN LA COSTA ANDALUZA
}

\author{
Ojeda Zújar J. ${ }^{1}$, Díaz Cuevas M. P. ${ }^{2}$, Prieto Campos A. ${ }^{3}$ y Álvarez Francoso J. I ${ }^{4}$ \\ Grupo de Investigación: Ordenación Litoral y Tecnologías de Información Territorial. ${ }^{5}$ \\ Departamento de Geografía Física y Análisis Geográfico Regional. \\ Universidad de Sevilla.
}

\section{RESUMEN}

Presente en numerosas bases de datos geográficas y cartografía oficial, la línea de costa constituye un elemento fundamental en los procesos de análisis, gestión y planificación de la zona costera, si bien su levantamiento a escalas de detalle y su posterior utilización requiere de una precisa delimitación geométrica y del establecimiento de criterios rigurosos para su definición. Éstos últimos deben ser seleccionados con una clara intencionalidad aplicada de acuerdo a su uso posterior. En este sentido, el uso de los Sistemas de Información Geográfica, las bases de datos espaciales y un adecuado modelo de datos, resultan elementos clave para el levantamiento de líneas de costa, así como para los análisis posteriores realizados sobre ellas.

En este trabajo se presenta, en primer lugar, la metodología utilizada para la definición geométrica de la línea de costa en Andalucía a una escala detallada (1:2.500) sobre ortofotos y ortoimágenes de resolución métrica y submétrica y el modelo de datos que soporta cada tramo topológicamente independiente digitalizado. Tras su digitalización geométrica e integración en una base de datos espacial (PostGres/PostGis) se utilizan las capacidades analíticas de las TIG para la construcción de diferentes indicadores utilizados en su caracterización fisiográfica, estado, seguimiento y evaluación ambiental en la costa andaluza.

Palabras clave: línea de costa, sistemas de información geográfica, geodatabase, modelos de datos, indicadores costeros.

\section{ABSTRACT}

\section{Coastline and geographical information systems: data model for the andalusian coast characterization and coastal indicators estimation}

The coastline is a key element for coastal planning and management, however, its digitalisation at fine scales requires a precise geometric delimitation and a rigorous criteria establishment for its definition. The criteria selection should be based on the work purpose and future applications. In this sense, Geographic Information System (GIS) techniques, spatial databases and an appropriate data model for the database design are key elements that enable establishing a work flow for coastlines digitalisation and subsequent analyses.

This paper presents a methodology for the Andalusia coastline definition and positioning at fine scale (1:2.500) using specific criteria for the coastline digitalisation and a data model for the spatial database design. The digitalisation and data entry in the database were carried out through ortho-photos

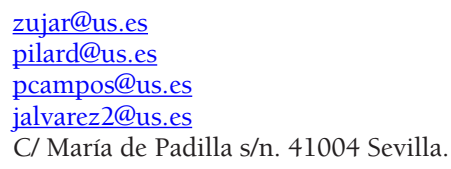


photointerpretation. After the digitalisation process, the data were stored in a spatial database (Postgres / PostGIS) for maximising the analytical capabilities, which has enabled indicators estimation for physiographic characterisation and environmental assessment for instance.

Keywords: coastline, Geographical Information System, geodatabase, data model, coastal indicators.

\section{INTRODUCCIÓN}

Hoy día, cualquier estudio centrado en la zona costera independientemente de cual sea su objetivo, requiere de la existencia de una potente información de detalle. Esta reflexión, a pesar de ser incorporada en numerosos documentos de planificación, se ve confrontada por la ausencia a veces inexplicable de información sobre litoral y medio marino a escalas de detalle (Díaz et al., 2012). En este sentido, la línea de costa constituye una referencia inexcusable para la planificación ambiental y territorial de estas zonas, así como para una gestión integrada de éstas, pues permite constituir indicadores de estado y seguimiento ambiental y, como tal, es frecuente encontrarla, en la actualidad, en los resultados de proyectos y estudios que incluyen del cálculo de indicadores sobre ella (EUROSION, Greenpeace, 2010, Estrategia de sostenibilidad de la costa, etc.).

Aunque su definición como elemento de contacto entre la superficie emergida y la oceánica (Dolan et al., 1980) está consensuada, su delimitación supone un gran desafío dada la gran dinamicidad natural de este límite, que además depende de la elección de criterios específicos para su delineación, definidos en función del objetivo perseguido, la resolución espacial, la fuente de información utilizada o del sistema de digitalización empleado (Ojeda, 2000, Fletcher et al., 2003, Boak and Turner, 2005, Hughes et al., 2006), siendo necesario mostrar, para su definición, una clara intencionalidad aplicada.

Así por ejemplo, si se pretende obtener información que resulte de utilidad para la gestión de la zona costera andaluza, se hace imprescindible una delineación de la línea de costa atendiendo a criterios adecuados desde un punto de vista fisiográfico, entendida ésta como el segmento lineal que recorre el margen del litoral andaluz en contacto con el mar, desde su inicio en Portugal hasta su extremo oriental en Murcia. Esta línea de costa alcanzará longitudes considerables al introducirse por los márgenes de los canales de marea y estuarios, y deberá delimitar detalladamente determinadas infraestructuras costeras presentes en ella (puertos, diques, espigones, etc.). Por otra parte, si se pretende utilizar ésta para el cálculo de indicadores y estadísticas oficiales, la definición de la línea, se suele asociar a lo que se entiende comúnmente por "frente costero expuesto", y evitará su recorrido detallado por marismas y estuarios, así como los contornos de puertos e infraestructuras costeras que penetran en el mar (Díaz et al., 2012).

Dado lo anterior, el desafío, entonces, es desarrollar una metodología suficientemente robusta y repetible para permitir de la manera más sencilla y eficaz la digitalización de este límite según los criterios elegidos. En este sentido, el uso de de un adecuado "modelo de datos" para su incorporación en Gestores de Bases de Datos es esencial y facilitará posteriormente las capacidades analíticas de los SIG (Thieler, et al., 1994, Brown, 2006; Guariglia et al., 2006; Rodríguez et al., 2009; Pian et al., 2011). Sin embargo, la definición de la zona de transición costera en modelos de datos que soporten información geográfica (ráster o vectorial) se ve especialmente dificultada por el carácter de interfase natural de ésta (atmósfera, litosfera, hidrosfera) y su alto dinamismo a escalas espacio-temporales muy cortas.

\section{OBJETIVOS}

El objetivo de este artículo, se centra en presentar los resultados del uso de una delimitación multifuncional de la línea de costa de Andalucía a escala de detalle (1:2.500), que incorpora tres conceptos de línea de costa diferentes, pero que mantienen para todas la misma escala y resolución a nivel espacial, así como el mismo modelo de datos en su caracterización alfanumérica. Este objetivo genérico se ve dificultado por la dimensión espacial $(916 \mathrm{Km}$.) y alta variabilidad fisiográfica de la costa de Andalucía.

El enorme esfuerzo ligado a la digitalización de una línea de costa para toda Andalucía desde la perspectiva geométrica (máxime si se espera su actualización continuada o incluso su utilización para fechas históricas pasadas) debe ir acompañado de la suficiente información temática (caracterización 
alfanumérica en un SIG) para cubrir el mayor número de aplicaciones futuras. Entre ellas destacan la generación de indicadores, su utilización para los cálculos de tasas de erosión o su utilización para la evaluación de un recurso turístico esencial como es la capacidad de carga de las playas. En este sentido, son objetivos específicos de este trabajo:

- La definición del modelo conceptual que soportarán todos los datos (geométricos y alfanuméricos) y su aplicaciones

- La definición de criterios claros para su definición y de digitalización a escalas de detalle.

- La extracción de algunos indicadores que, a modo de resultados preliminares, reflejen la versatilidad de la aproximación metodológica adoptada.

\section{3. ÁREA DE ESTUDIO}

La costa de Andalucía se extiende a lo largo de $916 \mathrm{Km}$. presentando dos tramos claramente diferenciados: un sector occidental hasta el Estrecho de Gibraltar, expuesto a un importante oleaje de fondo del océano Atlántico ( $f e t c h)$, con un carácter mesomareal y donde predominan las formaciones arenosas, estuarios y marismas mareales; y otro sector oriental (Estrecho de Gibraltar-Almería), expuesto al oleaje del mar Mediterráneo, micromareal y con una mayor presencia de costas rocosas y acantiladas, junto a playas, deltas y albuferas.

Figura 1. Área de estudio.

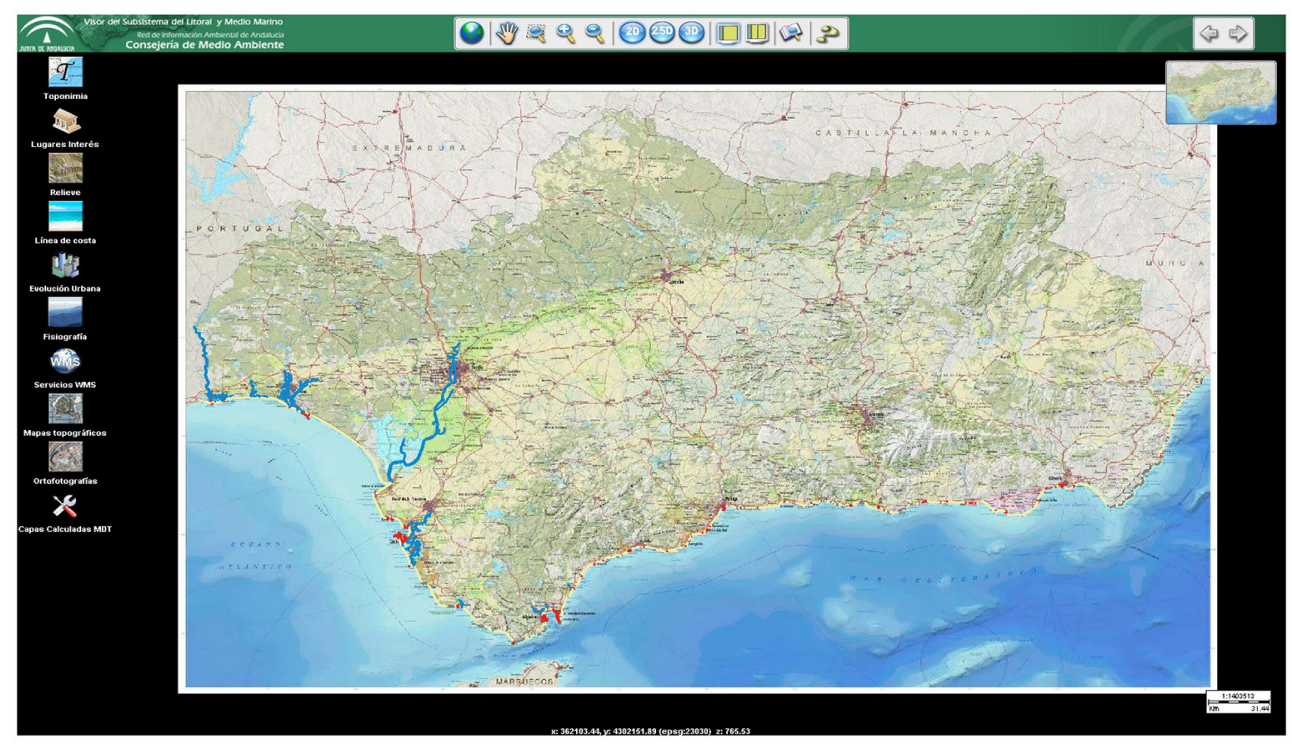

Fuente: Imagen del visor web tridimensional de Grupo de Investigación al que pertenecen los autores

Debido a su singularidad y peculiaridad al compartir una fachada costera abierta al Atlántico con una dinámica mesomareal y otra mediterránea con una dinámica micromareal, desde la perspectiva científica, estos dos ámbitos bien diferenciados han sido objeto de diferentes estudios por parte de diferentes investigadores e instituciones que han permitido tener una conocimiento bastante detallado de sus características físico-naturales y su evolución reciente (Viciana, 1998, Zazo y Goy, 2000; Ojeda, et al., 2002, Ojeda, 2003, Ojeda, 2005a, Del Río, 2007 entre otros), aunque parciales desde la perspectiva espacial. Todos ellos han proporcionado un conjunto considerable de información.

La Comunidad autónoma ha realizado amplios esfuerzos por generar información detallada del ámbito litoral. Destaca la definición geométrica de su línea de costa a una escala detallada (1:2.500) para varios años por el Grupo de Investigación al que pertenecen los autores, que será presentada a continuación y su inclusión en el Subsistema de Información del litoral y Medio Marino (http://www.juntadeandalucia. es/medioambiente/site/rediam/), proyecto impulsado y financiado por la Consejería de Medio Ambiente de la Junta de Andalucía (Ojeda (coord.), 2005b) con una importante contribución de otras consejerías. Estos trabajos previos están en la base de la propuesta metodológica presentada en este artículo. 


\section{METODOLOGÍA.}

\subsection{Modelo de datos: atributos alfanuméricos y tablas.}

Como en cualquier sistema de información complejo y de cierta entidad, los procesos de producción y levantamiento de la información y el de su posterior explotación persiguen, hasta cierto punto, objetivos diferentes (Ojeda et al., 2010). Es por ello que resulta conveniente reflejar que en esta propuesta metodológica se han utilizados dos modelos de datos diferentes: uno vinculado al proceso de producción diseñado para hacer la digitalización lo más ágil posible optimizando la labor del digitalizador, evitando posibles errores en la digitalización mediante el uso de una base de datos espacial o geodatabase, relaciones topológicas y la definición de dominios o subtipos; otro más complejo, el modelo de explotación, perfectamente normalizado, y diseñado para maximizar la capacidad analítica sobre los datos que se introducen en él a partir del modelo de producción, evitando la redundancia y la falta de atomización de los mismos. Este modelo final de explotación reside en un Gestor de Bases de Datos Espacial de código abierto: Postgres/PostGis.

Los datos del modelo de datos de producción, son tratados posteriormente, por un procedimiento en la base de datos (programado en PL/SQL o PL/PgSQL) que carga la información, de forma controlada, normalizada y detectando errores (con lo cual sirve de mecanismo de control de calidad) en el modelo de explotación. En este apartado del modelo de producción destacan las herramientas ESRI, ya que éstas proporcionan un marco inmejorable para la edición rápida y eficaz de datos. Ello hace que el proceso de digitalización sea ágil. El modelo de producción, por tanto, se basa en muy pocas tablas; de hecho, lo deseable es que el digitalizador sólo tenga que utilizar una en su tarea. Es sin duda el escenario de digitalización más ágil, ya que el digitalizador no tiene que estar introduciendo claves en las tablas, navegando por la relaciones de las mismas o rellenando formularios, concentrando su atención en la interpretación e introducción de datos en dicha tabla única.

El modelo de explotación (Figura 2), por contraposición al modelo de producción, si es un modelo complejo perfectamente normalizado para maximizar la capacidad analítica sobre los datos. El modelo de explotación es, como su propio nombre indica, el lugar donde los técnicos e investigadores, extraen productos geográficos y estadísticos de consumo final de la información que contiene. De hecho, de él se deriva un nuevo modelo de producción y, pensando en la necesidad de actualizaciones continuas, este último se retroalimenta con nuevos datos.

Figura 2. Modelo de datos de Explotación de la Línea de Costa.

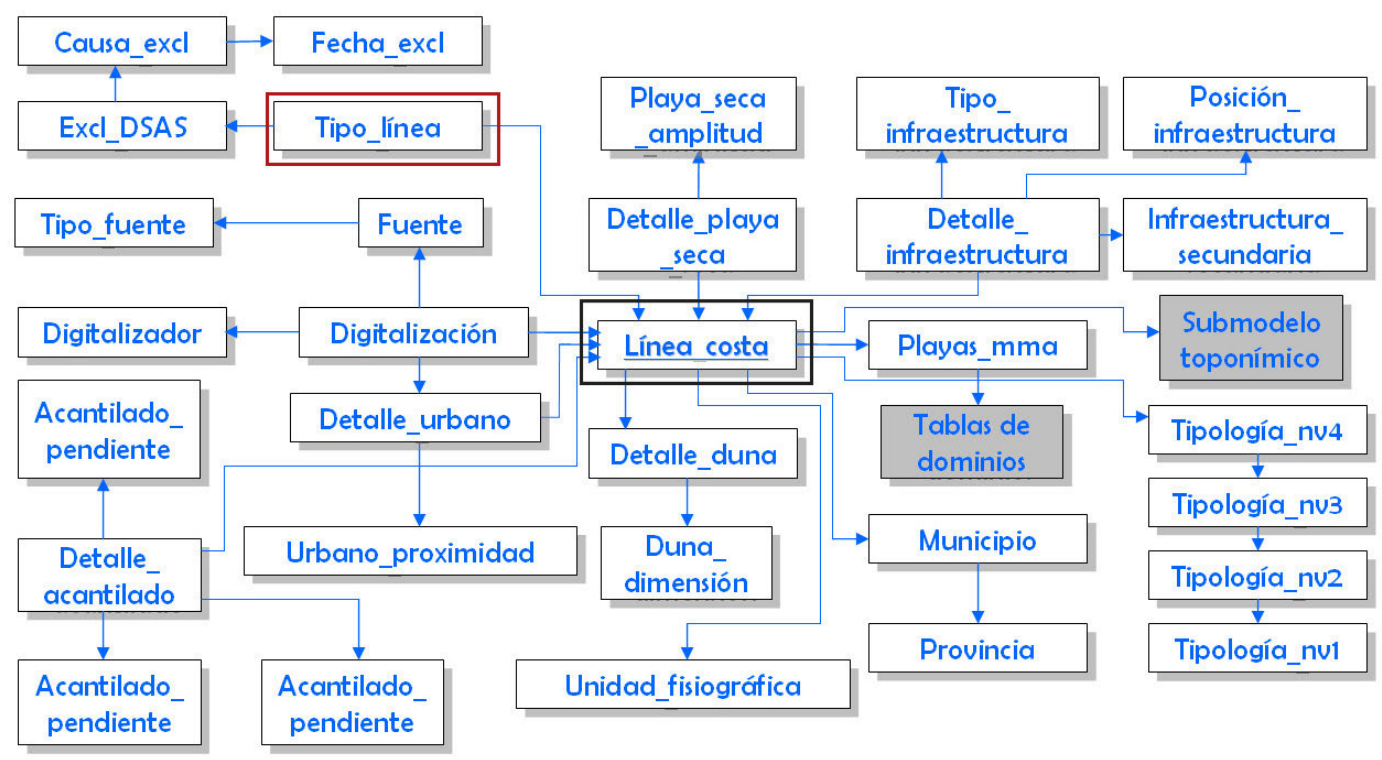

Elaboración propia. 
El objeto principal de este modelo de datos de explotación para la línea de costa andaluza es la tabla línea_costa, que recoge el inventario exhaustivo de tramos de línea de costa digitalizados topológicamente independientes, que comparten todos los atributos temáticos de las diferentes tablas relacionales del modelo. La segmentación de la costa en tramos topológicamente independientes resulta frecuente y es aplicada a escalas detalladas para los objetivos de planificación y gestión de estos ámbitos (Hoozemans et al., 1993; Mangor, 2001; Vafedeis et al., 2008, y Casal et al., 2010).

Un conjunto de estas tablas relacionan este tramo costero con el proceso de digitalización (autor, fecha y fuente) y su caracterización toponímica. Otro conjunto de tablas relacionadas reflejan la clasificación fisiográfica, tipológica y jerárquica, del tramo topológicamente independiente (tipologia_nvl, tipologia_nv2,...). La elección de una tipología jerárquica hace que las tipologías aumenten exponencialmente por la combinación de tipos básicos (playas con dunas y acantilado, por ejemplo) y la asignación del tipo en cada nivel excluyente. La imposibilidad de modelar en una estructura jerárquica una mayor caracterización de cada tipo (acantilados altos, acantilados bajos, o su naturaleza litológica en costas rocosas, por ejemplo) y su interés para el desarrollo de los indicadores ha obligado a modelar estos atributos en tablas externas a la que contiene la estructura tipológica jerárquica, ya que pueden coexistir varios sobre el mismo arco. Son las tablas relacionadas que enlazan con las tablas detalle_playa, detalle_duna, detalle_acantilado, detalle_infraestructuras y detalle_urbano.

Este modelo, fue diseñado por primera vez para el Subsistema Litoral y Medio Marino de la Red de Información Ambiental (REDIAM) de la Consejería de Agricultura, Pesca y Medio Ambiente de la Junta de Andalucía en 2007, si bien ha ido incorporando mejoras en esta última etapa al ser integrado y mejorado en diferentes proyectos de investigación. En el nuevo modelo presentado posee especial relevancia la incorporación de una nueva tabla relacional (tipo_linea) que permite mediante una codificación específica la definición de una línea de costa multifuncional cuya caracterización constituye el objetivo básico de esta comunicación.

\subsection{Fuentes, criterios de definición de línea de costa y procesos de digitalización.}

El levantamiento de la información relativa a las línea de costa a escala de detalle (1:2.500) se ha realizado mediante la digitalización de ésta a partir de la Ortofotografía de Andalucía de 2008/2009 (resolución espacial de 0,5 m.) y la ortoimagen QuickBird de 2005 (0,75 m.). El sistema de referencia de coordenadas utilizado ha sido ETRS89 UTM30N para respetar el Sistema de Referencia de Coordenadas de la fuente original de mayor resolución espacial, siendo el oficial en España desde 2007.

El levantamiento de la línea de costa ha sido realizada por un solo digitalizador, un solo experto temático, con el fin de disminuir al máximo posible la subjetividad inevitable en el proceso de fotointerpretación y siempre a una única escala (1:2.500), para garantizar la coherencia geométrica.

Los criterios de fotointerpretación utilizados (elementos clave para su posterior uso), a diferencia de otras líneas de costa "oficiales" que utilizan criterios geométricos (curva de nivel $0 \mathrm{~m}$.) sin una base ecológica, se definen por características geomorfológicas/fisiográficas diferentes según se trate de formaciones sedimentarias expuestas, costas rocosas, zonas marismeñas/estuarinas protegidas del oleaje o infraestructuras antrópicas.

- Estuarios y red mareal: definida como el límite de la marea más baja reconocible entre todas las ortofotos disponibles, representaría la línea más próxima al "0 hidrográfico" (no disponible a estas escalas para toda la costa andaluza). Su interés reside en que es necesaria para extraer la superficie intermareal, elemento que puede ser utilizado para diversos indicadores ambientales.

- Costas rocosas conectadas directamente con el mar: definidas a partir de la base de los acantilados o el límite de afloramientos rocosos. El hecho de que, en algunos tramos de acantilados altos en Andalucía se hayan observado desplazamientos geométricos debido al abatimiento del relieve que no ha podido corregir el MDE utilizado en la producción de la ortofotografia, así como su relativa permanencia temporal, es la causa que justifica la utilización de la imagen Quick Bird (2005) sólo en estos sectores. La altura de la órbita de la plataforma espacial que embarca a este sensor minimiza este efecto y su resolución espacial es parecida a la de la ortofotografía de 2008-2009. 
- Infraestructuras en contacto directo con el mar: definidas como el límite externo de las infraestructuras.

- Playas: Se trata del elemento más dinámico y de mayor interés aplicado (uso turístico, riesgos de erosión, etc.). Por ello se han utilizado dos criterios diferentes para su digitalización (Figura 3), es decir, se digitalizan dos líneas diferentes. La primera (Figura 3a), definida por la última marca húmeda de la marea sobre el perfil de la playa. Este límite define el límite exterior de la "playa seca" (backshore) en la fecha de la fuente de referencia y es la utilizada genéricamente (estadísticas, gestión, etc.). La segunda (Figura 3b) está orientada a la extracción de indicadores basados en tasas de erosión, por lo que se define por el contacto interno de la "playa seca" (backshore) y la duna costera (foredune) cuando está presente, la base de acantilados o el contacto con infraestructuras (paseo marítimo). Su carácter mas estable hace que sea la elegida en la mayor parte de los estudios evolutivos a largo plazo (Ojeda et al., 2000; Marcel J.F. et al., 2002; Pajack et al., 2002, Johannessen et al., 2003 y Ojeda et al., 2010). La distancia entre ambas; Figura 3c (que representa la superficie entre el límite interior y exterior de la playa seca) resulta, por otra parte, un elemento fundamental para el cálculo de la playa útil, de gran importancia para la construcción de numerosos indicadores (indicadores de capacidad de carga turística, de vulnerabilidad de las playas ante una potencial subida del nivel del mar, etc.).

Figura 3. Criterios para la digitalización de la playa: a) Marca húmeda de la última marea alta, b) Límite interno de la playa seca, c) Superficie entre el límite interior y exterior de la playa seca.
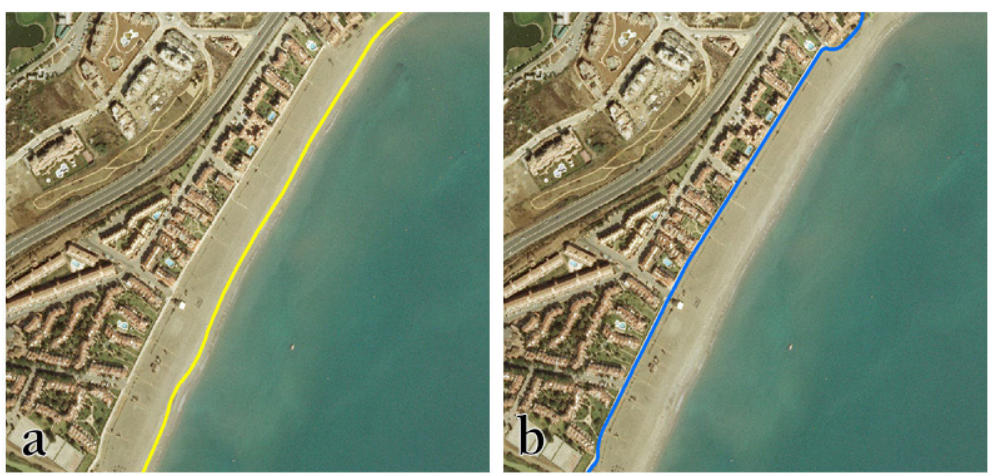

Elaboración propia

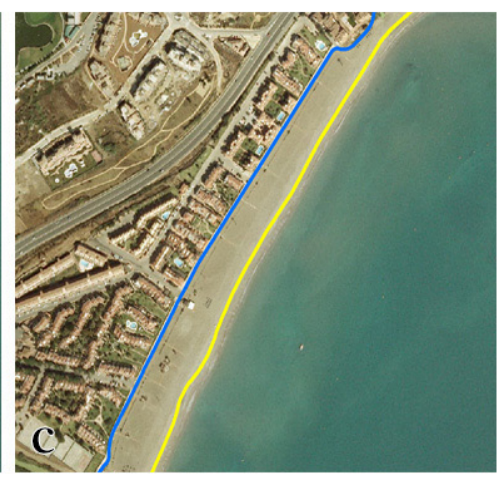

(C) $1 \%$

La conjunción de los elementos anteriores permite la extracción de dos tipos de línea de costa al máximo nivel de detalle espacial con objetivos aplicados diferentes que denominaremos:

- Línea de costa larga o definida fisiográficamente: en ella se incluye la digitalización detallada de elementos antrópicos del frente litoral (diques, espigones, puertos), así como estuarios y caños mareales cuya anchura en bajamar sea superior a 15 metros, la base de los acantilados y costas rocosas y el limite exterior de la playa seca al mayor nivel de detalle (Figura 4a).

- Línea para el cálculo de las tasas de erosión: en este caso solo se ven modificados los tramos de playa, donde la línea de costa queda fijada por contacto entre la playa alta (backshore) y la duna litoral (foredune). Ante la ausencia de duna litoral, se toma como válido el contacto superior de la playa alta con el primer elemento existente, ya sea natural (pie de acantilado) o antrópico (infraestructuras), (Figura 4c).

Estas dos líneas de costa soportarán el grueso de la aplicaciones tanto para la construcción de indicadores a escalas de detalle como para los análisis de procesos erosivos, estudios de impactos o gestión ambiental (por ejemplo si se necesitase saber la longitud de playas, diques, espigones o acantilados que se verían afectados por un vertido de petróleo) o la evaluación de la capacidad de carga turística de las playas.

Sin embargo, una gran parte estudios y análisis a partir de indicadores sobre la línea de costa suelen adoptar el concepto de "frente costero" para su cálculo, no contabilizando las zonas no expuestas al oleaje (no se digitalizan la red de caños mareales y estuarios, sino sólo su desembocadura) y no contabilizan 
tampoco el perímetro de las infraestructuras como puertos o espigones sino su sola presencia (la teórica línea de costa afectada). Por ello se han digitalizado estos dos elementos de forma simplificada y se incorporan estos tramos nuevos en el mismo modelo de datos con un código diferente en le tabla "tipo_linea". De esta forma es posible extraer una tercera línea de costa:

- Línea de costa corta o frente costero: Intenta definir el frente costero expuesto. Para ello, en la línea de costa larga se sustituyen: (i) elementos antrópicos como puertos y grandes espigones que penetran en las aguas marinas por línea costera original definida por la ortofoto de 1956; (ii) estuarios y caños mareales por una línea que cierra su desembocadura al llegar al frente costero (Figura 4b).

Figura 4. Representación de las líneas de costa larga (a), corta (b) y de erosión (c) en la Bahía de Algeciras (Cádiz).

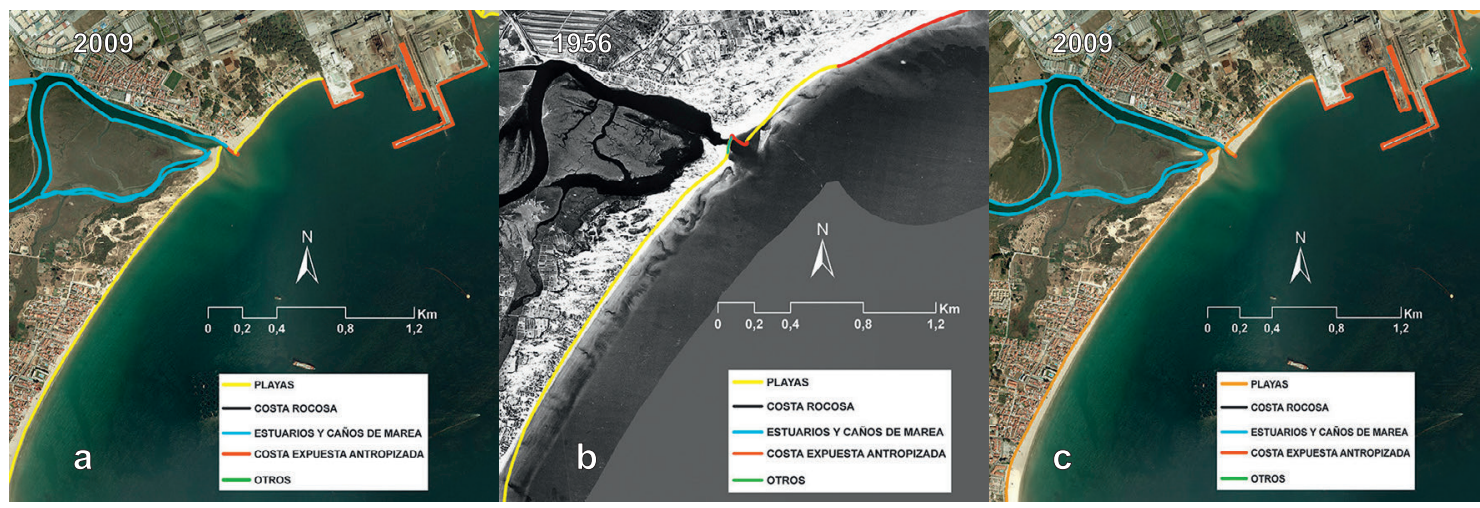

Elaboración propia

Estas tres líneas (larga, corta y erosión) se encuentran soportadas por el mismo modelo de datos. Para su posterior extracción a partir de sentencias SQL (structured query language) ha sido necesario la incorporación de una nueva tabla (tipo_linea) al modelo de datos que recoge la codificación del tipo (larga, corta o erosión) en función de su digitalización y utilización posterior.

\section{RESULTADOS}

El levantamiento de las líneas de costa, fotointerpretadas con criterios "geomorfológicos/fisiográficos" a escalas de detalle, junto a la versatilidad del modelo de explotación y producción empleado, así como su incorporación a una base de datos espacial, garantiza la fácil obtención de diferentes tipos de indicadores con sencillas sentencias espaciales SQL fácilmente reutilizables en actualizaciones futuras.

De esta forma, la longitud (parámetro esencial de muchos indicadores) de playas, estuarios, acantilados o infraestructuras para cualquier ámbito espacial (municipios, espacios protegidos, etc.) es una variable fácilmente extraíble de la base de datos y potencialmente utilizable para la generación de indicadores desde escalas autonómicas, a niveles intermedios (municipios, espacios protegidos, etc.) e, incluso, al nivel de playa individual (porcentaje de playa artificializada, porcentaje de dunas, etc.), algunos de ellos, ya presentados en una anterior publicación (Díaz et al., 2012).

Por otro lado, la utilización del modelo de datos, así como la delimitación multifuncional de la línea de costa, permite fácilmente el cálculo de otros indicadores que utilizan datos derivados directamente del modelo de datos pero que necesitan para su cálculo de un proceso de tratamiento posterior como son la definición de la playa útil, de especial importancia para el cálculo de la capacidad de carga turística previamente comentada, o las tasas de erosión.

\subsection{Sensibilidad de los indicadores de longitud y porcentaje de tipos fisiográficos de costa en función de la utilización de la "línea de costa larga" o "línea de costa corta"}

Producto de la digitalización y de la completa caracterización de la línea de costa realizada a escala de detalle 1:2.500, se obtiene una gran fragmentación longitudinal de la misma, conformada por 
5.858 segmentos para la línea de costa larga y 4.409 para línea corta, todos ellos de diverso tamaño y cuya suma determina una longitud total de $2.383 \mathrm{Km}$ y $916 \mathrm{Km}$, respectivamente.

Se observa (tabla 1), como la longitud de las playas, costa rocosa y de la tipología "otros" (esencialmente pequeños islotes rocosos), se mantiene prácticamente igual para ambas líneas mientras que, como era de esperar por la definición realizada de éstas, las mayores diferencias entre ellas se producen en los estuarios (más de $80 \mathrm{~km}$ en el caso del Guadalquivir hasta Sevilla y caños mareales, así como en la costa antropizada, que alcanzan valores considerables en el caso de la línea larga).

Tabla 1. Longitud de línea de costa en el nivel jerárquico 1.

\begin{tabular}{|l|c|c|c|c|}
\hline & $\begin{array}{c}\text { TOTAL LARGA } \\
(\mathrm{Km})\end{array}$ & $\begin{array}{c}\text { TOTAL LARGA } \\
(\%)\end{array}$ & $\begin{array}{c}\text { TOTAL CORTA } \\
(\mathrm{Km})\end{array}$ & $\begin{array}{c}\text { TOTAL CORTA } \\
(\%)\end{array}$ \\
\hline PLAYAS EXPUESTAS & 625,63 & 27,14 & 620,217 & 69,01 \\
\hline COSTA ROCOSA & 151,27 & 6,42 & 151,57 & 16,47 \\
\hline ESTUARIOS, CAÑOS DE MARISMA & $1.232,99$ & 51,41 & 25,57 & 2,79 \\
\hline COSTA EXPUESTA ANTROPIZADA & 365,08 & 14,69 & 111,69 & 10,85 \\
\hline OTROS & 7,75 & 0,34 & 7,81 & 0,88 \\
\hline & 2382,99 & 100 & 916,7 & 100 \\
\hline
\end{tabular}

Elaboración propia.

Sin embargo, en el caso de las playas, las diferencias entre ambas líneas se hacen patentes solo cuando se usan indicadores en términos relativos ya que al estar las playas siempre ubicadas en la zona expuesta del frente costero presentan en ambas la misma longitud (figura 5 a y b). Este indicador refleja que las playas están presentes casi en toda la costa andaluza, incluso en los sectores donde, por la proximidad de las sierras béticas, deberían ser más casas. Esto es debido a la gran aportación sedimentaria de los cauces que avenan hacia el Mediterráneo por las características de sus cuencas (elevada pendiente media) y régimen hidrológico con eventos de precipitación torrencial que favorecen la movilización de sedimentos hacia la costa y la construcción de playas junto a planicies litorales.

Figura 5. Detalle de la caracterización de las playas en la línea larga (a) y línea corta (b).

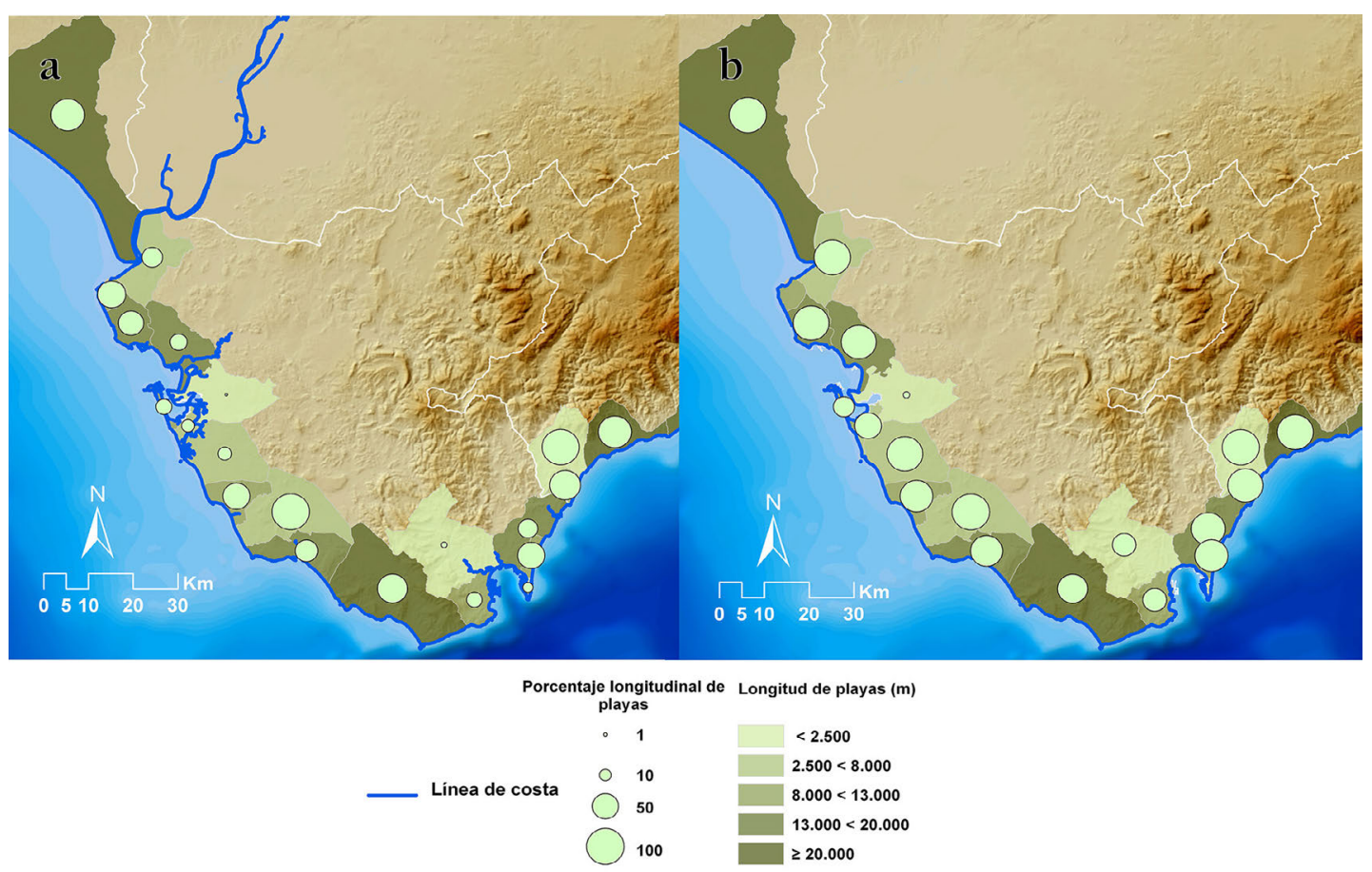

Elaboración propia 
Cuando se utilizan indicadores relativos (porcentajes), la utilización de una costa u otra devuelve valores porcentuales muy diferentes para cada municipio. Este hecho es más evidente en aquellos municipios donde la presencia de marismas y estuarios hace aumentar significativamente la longitud de costa municipal de referencia para la construcción del indicador (Huelva o Ayamonte) o en aquellos donde se ubican las grandes infraestructuras portuarias (Málaga, Bahía de Algeciras). Estos resultados evidencian la necesidad de precisar detalladamente las fuentes y criterios utilizados los indicadores de este tipo y establecer comparaciones solo con indicadores construidos de forma homogénea.

\subsection{Generación de un indicador de sensibilidad a la pérdida de las playas andaluzas como recurso turístico utilizando la "línea de costa larga" y la "línea de costa erosión".}

En la actualidad son varias las publicaciones científicas existentes que tratan el origen, evolución, estado y seguimiento de la costa andaluza, si bien suelen aplicarse a sectores concretos o ámbitos espaciales y fisiográficos diferentes, siendo compleja su integración. En el caso de las playas, elemento muy dinámico y de gran interés aplicado, su función como zona de esparcimiento y uso público y su grado de naturalidad suele ser medido mediante indicadores relacionados con el equipamiento público, la accesibilidad, la calidad de las aguas, etc. El análisis de su función como recurso turístico requiere también del levantamiento de información que permita tener definida perfectamente la playa útil desde el punto de vista turístico (playa seca) y su estabilidad (vinculada a los procesos erosivos) o persistencia temporal (ligada a la disponibilidad de reservas sedimentaria como las dunas costeras). En este sentido se utilizarán la línea de costa larga y la de erosión, junto con los atributos del modelo de datos, para definir un indicador de sensibilidad a las pérdidas de las playas como recurso turístico. Este indicador utiliza tres variables accesibles a escala de detalle, que han sido derivadas de las "líneas de costa" previamente generadas mediante las capacidades analíticas de los SIG.

\subsubsection{Calculo de las variables.}

La construcción del índice expresivo de la sensibilidad a la pérdida de las playas andaluzas como recurso turístico se ha realizado a partir del cálculo e integración de cuatro variables: anchura de la playa útil, presencia de dunas costeras, tasas de erosión históricas negativas y tasas de erosión históricas positivas.

- La anchura de la playa útil, figura 6, (relacionada con la superficie disponible como recurso turístico) se extraerá calculando la distancia entre la línea de costa larga y la de erosión que definen el límite externo e interno de la playa seca según el modelo de datos. A mayor anchura, menor sensibilidad de la playa a su pérdida como recurso turístico.

Figura 6. Izquierda: unidades fisiográficas que conforman la playa útil (playa natural). Derecha: tasa de anchura de la playa disponible por tramos individualizados de playa.
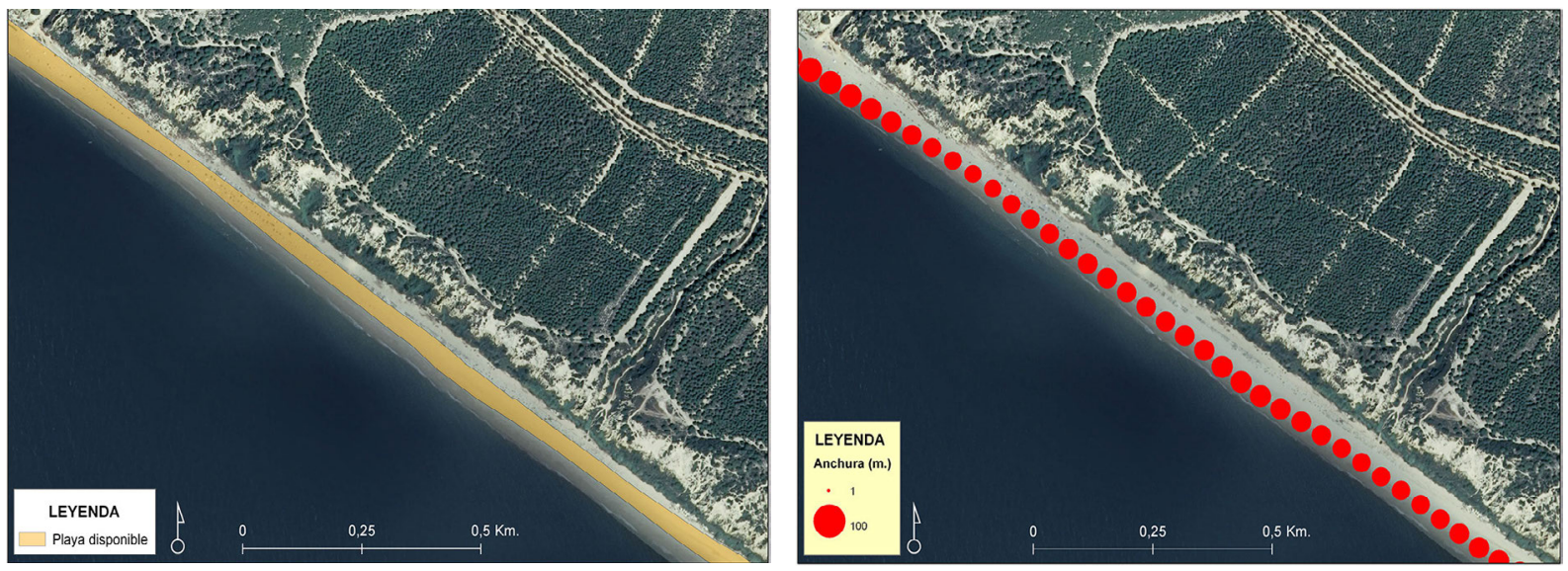

Elaboración propia 
- La presencia de dunas costeras: se extraerá de la tabla de datos asociada a cada tramo de playa: "duna_detalle" (figura 7). Esta incorpora en el proceso de fotointerpretación la presencia de dunas costeras en conexión con la playa actual y una estimación de su extensión hacia el interior, caracterizándolas según su extensión (extensa $>130 \mathrm{~m}$; media 30-130 m y pequeña $<30 \mathrm{~m}$ ). En este caso, la presencia de dunas costeras garantiza la persistencia de la playa aún cuando se vea sometida a procesos erosivos, aunque conlleve el consiguiente retranqueo de su posición original. En este sentido, a mayor extensión de dunas, menor sensibilidad de la playa a su pérdida como recurso turístico.

Figura 7. Digitalización de la duna.

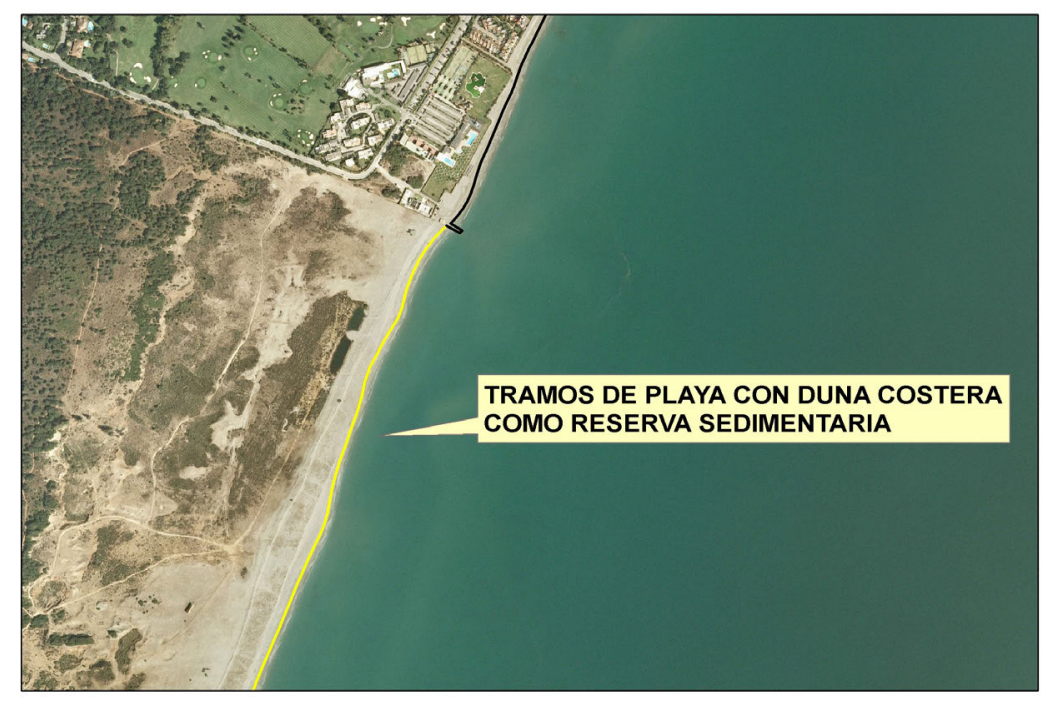

Elaboración propia.

- Las tasas de erosión históricas (figura 8), que se extraerán de la "desactualización" geométrica de la línea de costa de erosión más reciente (2009) a otras fechas para las que existen disponibilidad de ortofotos $(1956,1979,2007)$ y que ha sido objeto de varios trabajos publicados (Himmelstoss et al., 2009, Prieto et al., 2009, Prieto, 2012). Con estas líneas se procede al cálculo de las tasas de erosión anuales utilizando la herramienta DSAS, desarrollada por el Servicio Geológico de Estados Unidos e integrada en ArcGis. Esta permite su calculo para transectos equidistantes que genera el propio programa a petición del usuario y realiza el cálculo de la tasas de erosión (metros/año). A mayor tasa de erosión mayor sensibilidad de la playa a su pérdida como recurso turístico.

Figura 8. Tasas de erosión para el Delta del Albuñol y Huarea
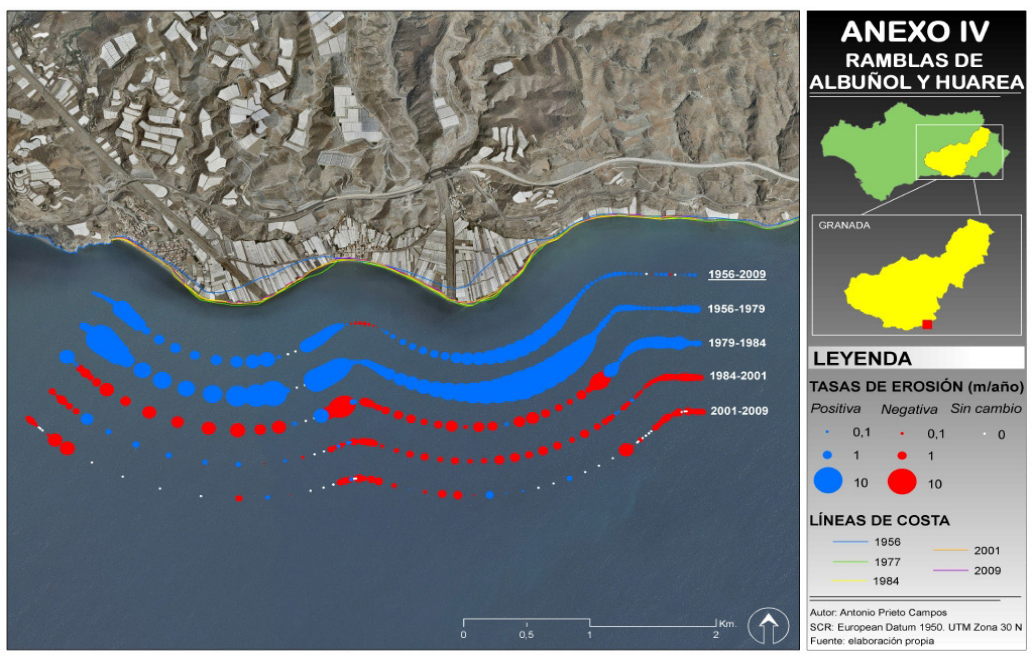

Fuente: Prieto, 2009. 
Esta misma herramienta permite cuantificar en los mismos transeptos la anchura de la playa útil si realizamos el análisis con la línea de costa larga y la costa erosión de la fecha 2009 y podemos extraer a los mismos transectos la extensión de la duna costera con un "join espacial". Esta flexibilidad en la generación de la equidistancia de los transectos nos permite asociar las cuatro variables necesarias para la generación del indicador a diferentes escalas según los objetivos y asociar las cuatro variables a puntos equidistantes tras la intersección de los transectos con la línea de costa más actual (2009).

\subsubsection{Formulación del indicador.}

Una vez calculadas las variables se ha procedido a la construcción del indicador a partir de la agregación de éstas. Previamente, las variables han sido normalizadas, con el objeto de expresarlas en unidades similares. Para tal fin los valores de éstas se reescalan entre 0 y 1 , mediante un reescalado lineal que asigna un 1 al valor máximo de la serie y un 0 al valor mínimo.

$$
X_{\text {in }}=1-\frac{\left(X_{i}-X_{\max }\right)}{\left(X_{\text {min }}-X_{\text {max }}\right)}
$$

Donde:

$X_{\text {in }}=$ Valor normalizado de la variable

$X_{\mathrm{i}}=$ Valor de la variable

$X_{\max }=$ Valor máximo de la variable

$X_{\min }=$ Valor mínimo de la variable

La variable tasas de erosión, ha sido transformada en dos variables; una representativa de las playas que han experimentado un balance sedimentario positivo en el periodo1979-2009 y otra representativa de las playas que han experimentado erosión en el periodo 1979-2009. Una vez que todas las variables tienen la misma intencionalidad y sentido, se ha procedido a la integración de las cuatro variables normalizadas originando el índice sintético representativo de la sensibilidad a la pérdida de playas.

La expresión del indicador es la siguiente:

$$
S=(D+A+P)-E
$$

Donde;

$\mathrm{S}=$ Indicador.

$\mathrm{D}=$ Duna.

$A=$ Anchura de la playa o playa disponible.

$\mathrm{P}=$ Progradación

$\mathrm{E}=$ Erosión.

Es necesario mencionar, que aunque este índice proporciona resultados de forma numérica, estos no deben asociarse directamente a cambios físicos específicos, es decir, se trata de un indicador adimensional. Sin embargo, tiene la capacidad de mostrar, a escala detallada y para toda la costa andaluza, cómo los efectos combinados de la erosión, la anchura de la playa útil y la presencia o ausencia de dunas intensifican la sensibilidad de determinados espacios costeros a la pérdida de playas.

\subsubsection{Aplicación del indicador y resultados.}

El indicador ha sido representado utilizando como límites de clasificación los percentiles 25\%, 50\% y $75 \%$. Los resultados, arrojan valores entre $-0,56 \mathrm{y}+2,17$ (tabla 2.). 
Tabla 2. Clasificación del Índice de sensibilidad.

\begin{tabular}{|c|c|c|}
\hline CLASE & $\begin{array}{c}\text { SENSIBILIDAD } \\
\text { CLASIFICADA }\end{array}$ & VALOR DEL ÍNDICE \\
\hline Muy Alta & 1 & $(-0.56 ; 0.037]$ \\
\hline Alta & 2 & $(0.037 ; 0.166\}$ \\
\hline Media & 3 & $(0.166 ; 0.75]$ \\
\hline Baja & 4 & $(0.75 ; 2.17]$ \\
\hline
\end{tabular}

Elaboración propia

La Figura 9a, representa el 20\% de los valores más bajos alcanzados por el indicador, es decir; las zonas del litoral andaluz con mayor sensibilidad a la pérdida de las playas. Éstas se corresponden con aquéllas playas que poseen menor superficie de playa útil, mayores tasas de erosión y no poseen dunas costeras susceptibles de ser aprovechadas como reserva sedimentaria. Principalmente la mayor parte de ellas se localizan en el litoral mediterráneo, donde el aporte sedimentario es menor que en el litoral Atlántico y donde la presión antrópica es mucho más intensa. Esta intensificación antrópica (urbana y de infraestructuras costeras) ha provocado por un lado, un gran impacto sobre las dunas costeras, las cuales han desaparecido o han sido reducidas en gran medida, y por otro, una modificación en el balance sedimentario costero, induciendo un incremento en las tasas de erosión en determinados ámbitos.

La Figura 9b, muestra las zonas del litoral andaluz con menor sensibilidad a la pérdida de playas (20\% de los valores más altos alcanzados por el índice). Éstas se corresponden con aquéllas playas más anchas, que poseen dunas y un balance sedimentario positivo. Éstas se encuentran mayormente en el litoral atlántico, donde la aplicación de diferentes figuras de protección a determinados espacios coteros (Parque Nacional de Doñana, Parque Natural de Doñana, Paraje Natural Marismas del Odiel, Monumento Natural del Asperillo), ha favorecido un mayor grado de naturalidad, una menor presión antrópica y la preservación de la duna costera en muchos sectores. Este hecho se registra también en algunos enclaves del litoral mediterráneos, que se encuentran bajo alguna figura de protección (Parque Natural Cabo de Gata y Níjar, Paraje Natural Punta Entinas-Sabinar o Monumento Natural Dunas de Artola o Cabopino, en Marbella).

Figura 9. Representación de los mayores y menores valores de sensibilidad a la pérdida de las playas andaluzas como recurso turístico.

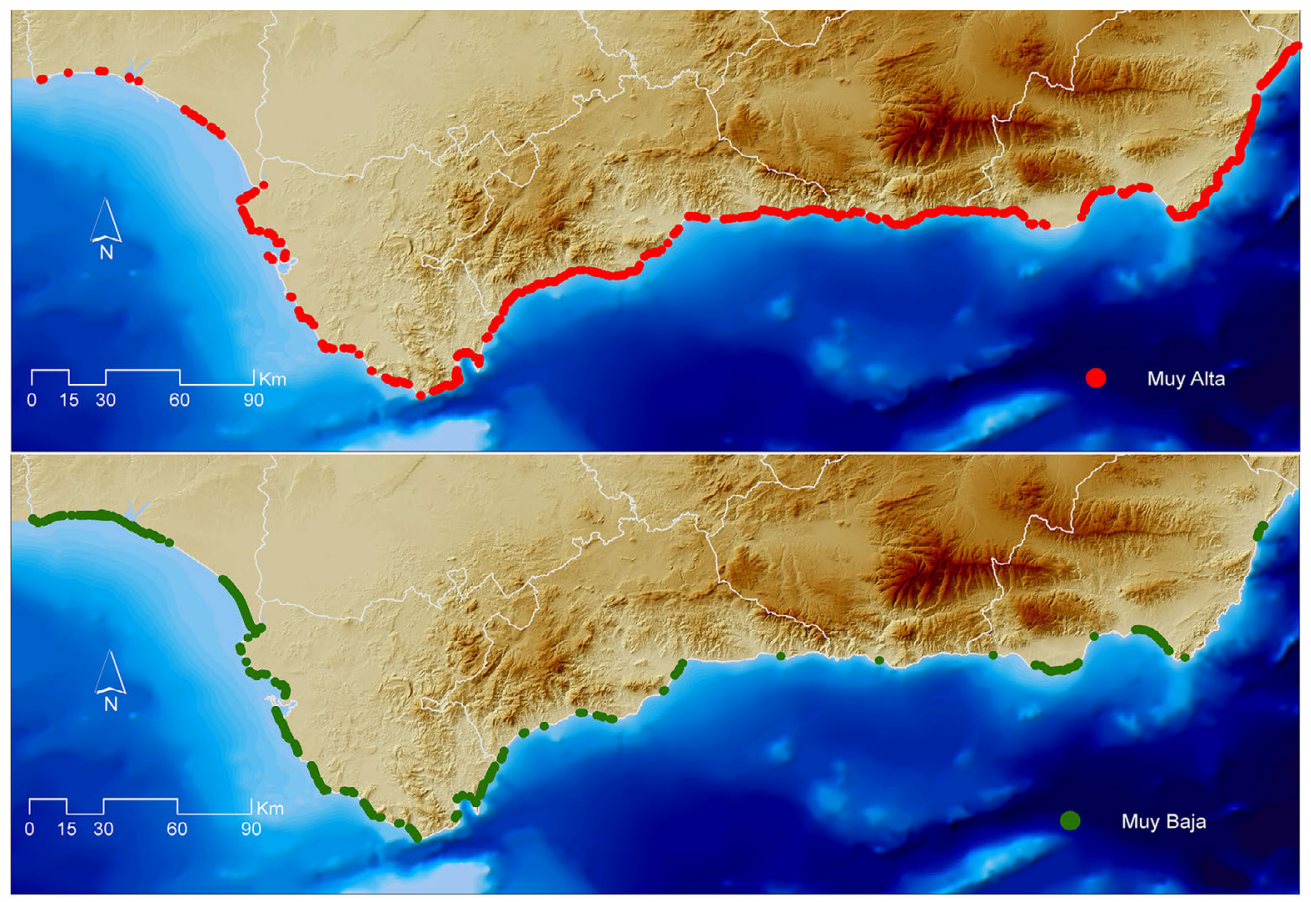

Elaboración propia. 
Al ser el índice generado a partir de geodatos, la utilización de las diferentes técnicas de análisis espacial facilitadas por los SIG, permiten la expresión de este de forma estadística (véase figura 10), asociando las variables a diferentes entidades (municipios, provincias, espacios protegidos, etc.).

Para la representación cartográfica del índice, (véase figura 11), se ha utilizado el método de representación utilizado por el Servicio Geológico de Estados Unidos (USGS) para el Índice de Vulnerabilidad Costera (CVI), que ha sido previamente utilizado por los autores en trabajos anteriores (Ojeda et al., 2009): así, para todas las variables y el cálculo total de sensibilidad, se adopta una representación de rectángulos paralelos a la costa, que pueden ser de diferente tamaño según la escala, utilizando las relaciones de cardinalidad entre "puntos originales" - asociados a tramos de $50 \mathrm{~m}$. de costa y "rectángulos de representación". Ello se realiza mediante la asignación del valor medio a los rectángulos de la representación cartográfica.

Figura 10. Presentación estadística de los resultados agregados a nivel provincial.

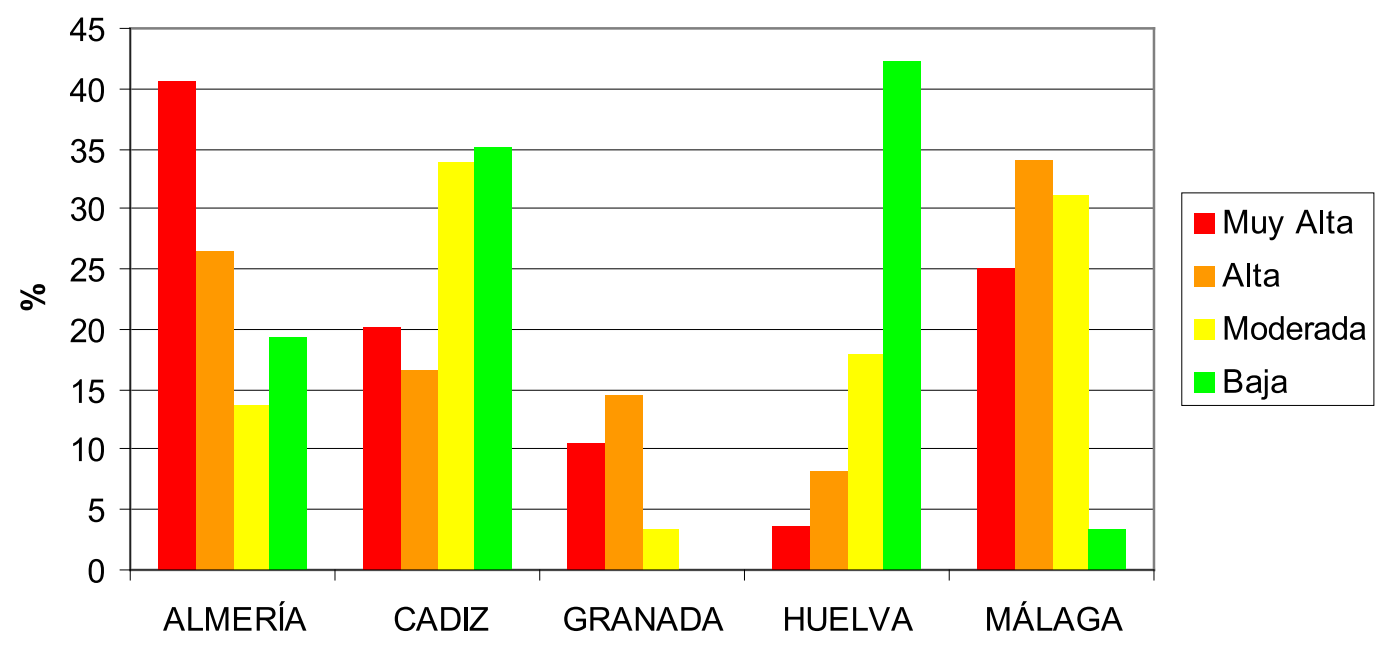

Elaboración propia.

Figura 11. Representación del Indicador de Sensibilidad a la pérdida de las playas andaluzas como recurso turístico y las variables explicativas.

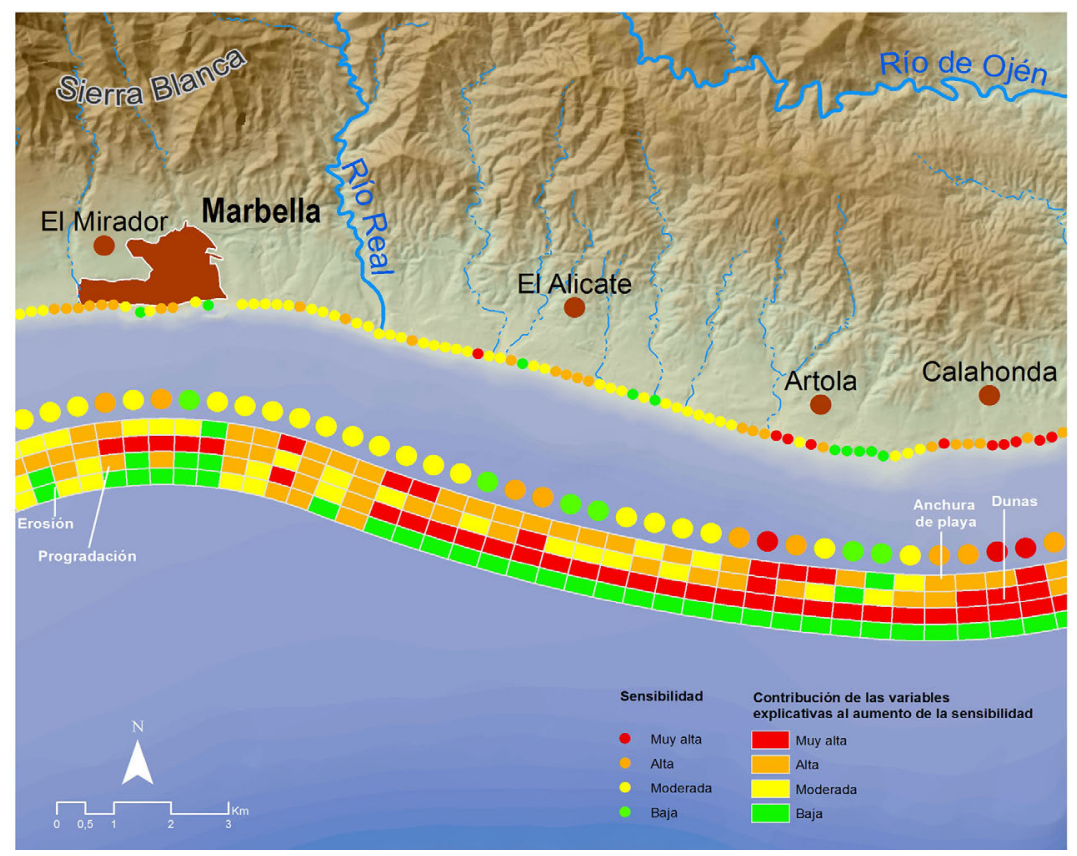

Elaboración propia. 


\section{CONCLUSIONES.}

El levantamiento de una línea de costa fotointerpretada en base a tres conceptos de línea de costa diferente a escalas de detalle, en base a criterios ecológicos/fisiográficos, unido a la versatilidad del modelo de datos conceptual empleado (a pesar de su coste y el esfuerzo exigido), garantizan la disponibilidad de un elemento esencial, de gran utilidad en los procesos de planificación y gestión ambiental de la costa de Andalucía y permiten al planificador y gestor un pormenorizado conocimiento de la fisiografía de la zona costera permitiendo su uso a diferentes escalas espaciales.

Por otro lado, su incorporación en una base de datos espacial facilita todas las labores de gestión y actualización de los mismos y garantiza la fácil obtención de diferentes tipos de indicadores, desde los que se extraen directamente de la base de datos a partir de sencillas sentencias SQL espaciales (la longitud de playas, estuarios o infraestructuras para cualquier ámbito espacial), a indicadores más complejos (sensibilidad a la perdida de playas como recurso turístico), que necesitan de un tratamiento posterior para su construcción y que resultan de gran interés para los investigadores y planificadores.

Los resultados de la aplicación de esta metodología, al ser generados a partir de una geodatabase y la utilización de las diferentes técnicas de análisis espacial que facilitan los Sistemas de Información Geográfica, pueden finalmente ser expresados tanto de forma estadística, asociándolos a diferentes entidades (municipios, provincias, espacios protegidos, etc.) como de forma cartográfica o alimentar a servidores de mapas con la construcción del servicios interoperables para su visualización o reutilización remota vía Internet.

Así, en todo el proceso el uso de las Tecnologías de la Información Geográfica, ha resultado un elemento fundamental para el desarrollo de una metodología eficiente, que permite de manera eficaz la disponibilidad de una línea de costa en Andalucía fácilmente actualizable, así como de comparables indicadores a escala detallada asociados a estas, algunos de ellos no calculados previamente para la totalidad de la costa andaluza.

\section{AGRADECIMIENTOS}

El presente trabajo se ha desarrollado dentro del proyecto correspondiente al Plan Nacional (CSO2010-15807) y del proyecto de excelencia de la Junta de Andalucía (RNM-6207): "Espacialización y difusión web datos de urbanización y fitodiversidad para la evaluación de la vulnerabilidad ante los procesos de inundación asociados a la subida del nivel del mar en Andalucía"

\section{BIBLIOGRAFÍA}

BOAK, E. H. y TURNER, I. L (2005): "Shoreline Definition and Detection: A Review", en Journal of Coastal Research, 1, pp. $688-703$.

BROWN, I. (2006): Modelling future landscape change on coastal floodplains using a rule-based GIS. Environmental Modelling Software. Elsevier, 21 (10), pp. 1479- 1490.

BURKE, L., Y. KURA, K. KASSEM, C. RAVENGA, M. SPALDING, and D. MCALLISTER (2001): Pilot Assessment of Global Ecosystems: Coastal Ecosystems. World Resources Institute (WRI). Washington D.C., 94 p.

DEL RÍO, L. (2007): Riesgos de erosión costera en el litoral atlántico gaditano. Tesis Doctoral. Universidad de Cádiz. 496 p.

DÍAZ, P., FERNÁNDEZ, M., PRIETO, A. y OJEDA, J. (2012): "La línea de costa como base para la generación de indicadores de estado y de seguimiento ambiental: modelo de datos y conceptos de líneas de costa en el litoral de Andalucía", en Tecnologías de la Información Geográfica en el contexto del Cambio Global, editado por CSIC y la Asociación de Geógrafos Españoles. Madrid, pp.35 - 44.

DOLAN, R., HAYDEN, B.P., MAY, P., and MAY, S.K. (1980): "The reliability of shoreline change measurements from aerial photographs", en Shore and Beach, 48(4), pp. 22 - 29. 
EUROPEAN COMISSION (2004): Coastal Erosion in Europe. Sediment and space for sustainability. Result from EUROSION Study, editado por P. Doody, M., Ferreira, S., Lombardo, et al., Netherlands.

FLETCHER, C.; ROONEY, J.; BARBEE, M.; LIM, S. y RICHMOND, B. M. (2003): "Mapping shoreline change using digital ortophotogrammetry on Maui, Hawaii", en Journal of Coastal Research SI 38, pp. 106- 124.

GREENPEACE, (2010): Destrucción a toda costa, Informe Greenpeace sobre la situación del litoral español. Madrid, $168 \mathrm{p}$.

GUARIGLIA, A., BOUNAMASSA, A., LOSURDO, A., SALADINO, R., TRIVIGNO, M. L., ZACCAGNINO, A. y COLANGELO, A. (2006): "A multisource approach for coastaline zapping and identification of shoreline changes", en Annals of Geophysics, 46, 1. pp. 295-304.

HIMMELSTOSS, E.A. (2009): "DSAS 4.0 Installation Instructions and User Guide", en Thieler, E. R., Himmelstoss, E.A., Zichichi, J. L., and Ergul, Ayhan. 2009 Digital Shoreline Analysis System (DSAS) version 4.0 - An ArcGIS extension for calculating shoreline change. U. S. Geological Survey Open-File Report 2008 - 1278.

HOOZEMANS, F. M. J, MARCHAND, M., and PENNEKAMP H.A. (1993): "A global vulnerability analysis, vulnerability assessments for population, coastal wetlands and rice production on a global scale, 2nd edn", en Delft Hydraulics and Rijkswaterstaat, Delft.

HUGHES, M., MCDOWELL, P.F. and MARCUS, A. (2006): "Acuraccy assestment of georectified aerial photographs: Implications for masuring lateral channel movement in a GIS" en Geomorphology 74, pp.1-16

JOHANNESSEN, J. y CHASE, M. (2003): "Coastal Processes, Historic Shoreline Change, and Sediment Distribution of Portage Bay, Lummi Indian Reservation, WA", en Puget sound reserarch conference, pp. 1-19.

MANGOR, K. (2001): Shoreline Management Guidelines. DHI Water \& Environment, 232 p.

STIVE, M., AARNINKOV, S., HAMM L., LARSON, M., WIJNBERG, R., NICHOLLS, R. and CAPOBIANCO, M. (2002): "Variability of shore and shoreline evolution", en Coastal Engineering 47, pp. 211 - 235.

MINISTERIO DE MEDIO AMBIENTE (2007): Estrategia de Sostenibilidad de la costa. Madrid, 23 p.

OJEDA, J.:

- (2000): "Métodos para el cálculo de la erosión costera. Revisión, tendencias y propuesta" en Boletín de la $A G E$ n $^{\circ} 30$, pp. $103-118$.

- (2003): "Las costas andaluzas", en Geografía de Andalucía. (Coord. Antonio López Ontiveros). Barcelona, Ariel, pp. 118 - 135.

- (2005a): "El mapa fisiográfico del litoral de Andalucía", en Atlas de Andalucía, Tomo II: Cartografía Ambiental. Consejería de Obras Públicas y Transporte y Consejería de Medio Ambiente. Junta de Andalucía. pp. 241 - 259 (5 hojas a escala 1:200.000).

- (2005b): Desarrollo del subsistema de información del litoral de Andalucía y generación de indicadores de seguimiento del medioambiente costero para el Centro Temático Europeo del Territorio. Consejería de Medio Ambiente. Junta de Andalucía.

OJEDA, J., BORGNIET, L., PÉREZ, A. y LODER, J. (2002): "Monitoring morphological changes along the coast of Huelva (SW Spain using soft-copy photogrammetry and GIS)", en Journal of Coastal Conservation, 8.1, pp. $69-76$.

OJEDA, J., ÁLVAREZ, J. I., MARTÍN, D. y FRAILE, P. (2009): "El uso de las TIG para el cálculo del índice de vulnerabilidad costera (CVI) ante una potencial subida del nivel del mar en la costa andaluza (España)", en GeoFocus, no 9, pp. 83 - 100.

OJEDA J., FERNÁNDEZ, M., PRIETO, A., PÉREZ, J. P. y VALLEJO, I. (2010): "Levantamiento de líneas de costa a escalas de detalle para el litoral de Andalucía: criterios, modelo de datos y explotación", en Tecnologías de la Información Geográfica: La Información Geográfica al servicio de los ciudadanos, editado por J. Ojeda, M.F. Pita e I. Vallejo. Sevilla, pp. 324-336. 
PAJAK, M.J. y LEATHERMAN, S.P. (2002): "The high water line as shoreline indicator", en Journal of Coastal Research, 18(2), pp. 329-337.

PIAN, S. and MENIER, D. (2011): "The use of a geodatabase to carry out a multivariate analysis of coastline variations at various time and space scales", en Journal of Coastal Research, 64, pp. 1722 - 1723.

PRIETO, A. (2009): Propuesta metodológica para el cálculo de tasas de erosión aplicada a los deltas mediterráneos andaluces (1956-2009). Departamento de Geografía Física y Análisis Geográfico Regional. Universidad de Sevilla. 109 pp. Inédito.

RODRÍGUEZ, I., MONTOYA, I., SÁNCHEZ, M.J. y CARREÑO, F. (2009): "Geographical Information Systems Applyed to Integrated Coastal Management”, en Geomorphology, 109, pp. 100 - 105.

THIELER, E. R. and DANFORTH, W.W. (1994): "Historical shoreline mapping (I): improving techniques and reducing positioning errors", en Journal of Coastal Research, 10, nº. 3, pp. 549-563.

VAFEIDIS, A. T., NICHOLLS, R.J., MCFADDEN, L., TOL, R. S.J., HINKEL, J., SPENCER, T., GRASHOFF, P.S., BOOT, G. and KLEIN, R.J. T. (2008): "A new global coastal database for impact and vulnerability analysis to sea-level rise", en Journal of Coastal Research, Vol.24 (4), pp. 917 - 924.

VICIANA MARTINEZ-LAGE, A. (1998): La erosión antrópica en las acumulaciones sedimentarias del litoral almeriense. Tesis Doctoral. Universidad de Granada.

ZAZO, C. y GOY, J. L. (2000): "Cambios eustáticos y climáticos durante el Cuaternario. Una síntesis sobre su registro en los litorales del sur y sureste de la Península, Islas Canarias y Baleares", en Geomofología Litoral. Procesos activos (Ed. J. R. de Andres y FJ. Gracia). ITGME. pp. 187-206. 